\title{
THE USING OF ADDITIVE MANUFACTURING FOR PROTOTYPE PRODUCTION OF MOULDS
}

\author{
Spânu Alina Rodica ${ }^{1}$, Victor Constantin ${ }^{2}$ \\ 1,2Politehnica University of Bucharest, Mechatronics and Precision Engineering Department, \\ Splaiul Independenţei, nr. 313, Bucharest, Romania \\ E-mail: spanu alina@yahoo.com
}

\begin{abstract}
The development of advanced applications regarding the additive manufacturing (AM) have become widely known starting with industrial applications and finishing with medicine devices. The paper presents a simple and very efficient way for manufacturing the moulds as prototypes that could be a cheaper, very accurate and more widely used solution for applying some type of new materials, beginning with the design of three-dimensional model for the core moulds without any supplementary equipments and classic technologies.
\end{abstract}

Keywords: Rapid Tooling, Fused Deposition Modeling, Moulds.

\section{Introduction}

During the new product development, the very short period for its market presentation is the main challenge facing the new technologies, which have been improved rapidly on the previous ones. Among these new technologies there are the AM (Additive Manufacturing) that afford the users the model manufacturing directly from Computer Aided Design implying a great reduction of manufacturing time, between three and nine times in comparison with the classic ones. The AM technologies are a new category of manufacturing a virtual model by using a family of special equipment. They have to add or glue together the material in successive layers as it is demanded and where it has to be done.

The advantages of these new technologies are related to the manufacturing of complex parts with spatial shapes that are impossible to attend with other technologies. Furthermore, the part complexity does not influence the price as it happens in some other situations.

The lately improvements of AM technologies are connected with IT, CAD and CAM upgrades, especially for the new materials that have played an important role in their development.The practices for new AM technology development have been extended from industrial field to medicine. A main part of this practice is known as Rapid Tooling, meaning some other tools that are made of other materials with other technologies, in order to increase the flexibility for small and medium production series. The flexibility idea is pointing out the short time of changing and adapting the tools with affordable costs, when we have to modify the needed product on the international market.

The flexible tool concept has some more meanings, outer the boundary of their flexibility regarding the manufactured product, having the following important features:

- there are tools or moulds required for small serie production;

- the flexible tools are linked normally with cheap prices;

- Meantime the idea of flexible tools is about the material type used for their manufacturing $[1][2][3][4]$.

\section{The Manufacturing Process}

The manufacturing moulding process requires a series of development stages when the steel or the alloy having liquid state is casted in a cavity in order to copy it by passing through a solidity state. A main part of the process is the model manufacturing that has to have physically the same shape as the part, which will be moulded and will give the external shape of the part. If the future part has some cavities, we have to use cores.

The models for steel moulding are divided in two categories: the permanent model that are used many times, and temporary models that are used for only one operation.

The permanent models are made of wood, steel, plastic. The temporary models are made of

From their manufacturing point of view, the models could be manufactured as following:

- handing manufacturing for the models made of wood;

- by cutting for steel models with regular shapes.

- by infusing or moulding for the fusible models

- manufacturing by additive material 
The using of additive manufacturing for prototype production of moulds

The introducing and development of AM technologies provided a cheaper, more rapid method and sometimes a higher accuracy way for manufacturing all model and core types with a wide range of material sort. We may start from their virtual 3D model without needing other equipment and classic technologies used before, so that it could be a solution for prototype or small series products.

The AM technologies are following some main stages as:

- The CAD design of the model that provides the entire description of the part, by using a CAD software, a scanning system and so on.

- The CAD model is transferred through the sectional processor;

- The triangles of the model that were prepared before are ready for sectioning and computation;

- The part manufacturing;

- During the cleaning and finishing stages, it has to be removed the auxiliary frames used for manufacturing or some other supplementary materials needed for the process in order to increase the dimensional accuracy and quality of the part.
The Fused Deposition Modelling is based on the material extrusion of the PLA (Poly Lactic Acid), ABS (Acylonitrile Butadiene Styrene) which is warmed to few degrees beneath its melting point. After that its diameter is reduced to $0.12-0.15 \mathrm{~mm}$ by extrusion into a deposition device placed in the XOY plane for implementing the section of virtual 3D model (fig. 1).

The key of the process consists of very accurate temperature control for material warming and maintaining during the deposition. The used material is PLA and the working temperature value is $215{ }^{\circ} \mathrm{C}$, so the material has a liquid state that affords its extrusion through a nozzle with very small dimension diameter. The nozzle needed to extrude the material together with the warmed head on which it is working could be displaced along the XOY plane by computer movement controlling.

The platform on which the part is put could be moved along the $\mathrm{OZ}$ axis, in vertical direction by using computer numerical control of the printer. For the mold described in the paper and presented in Fig. 2, we have manufactured a pair of half mold that have the dimensions presented in Fig. 1.

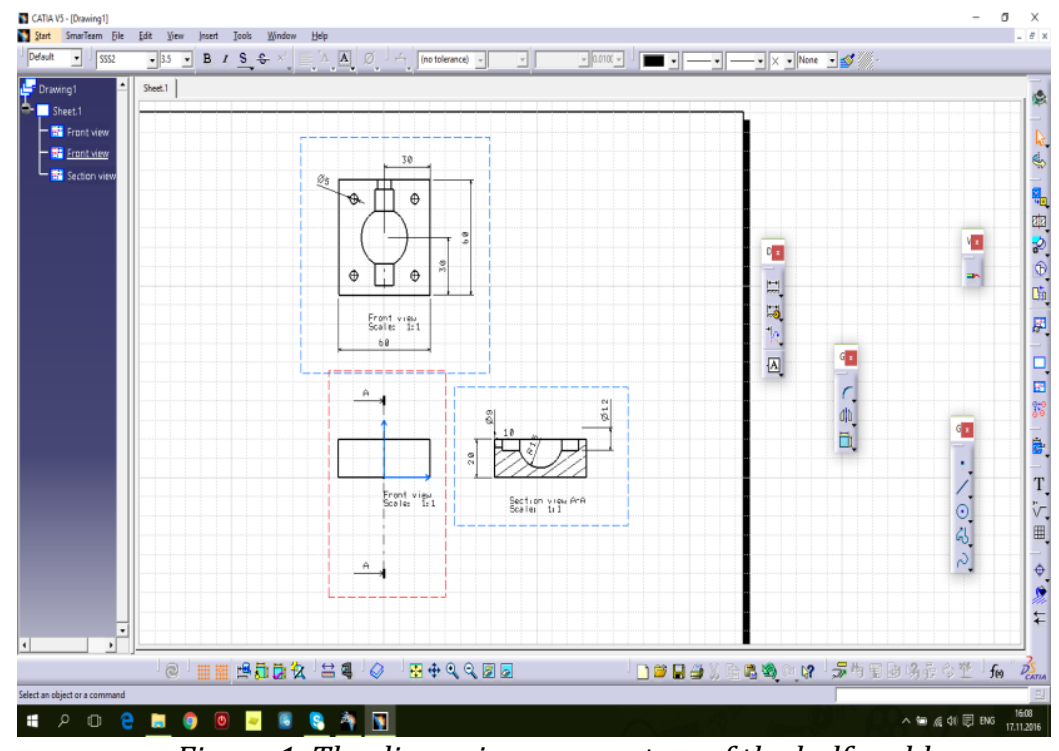

Figure 1. The dimension parameters of the half mold

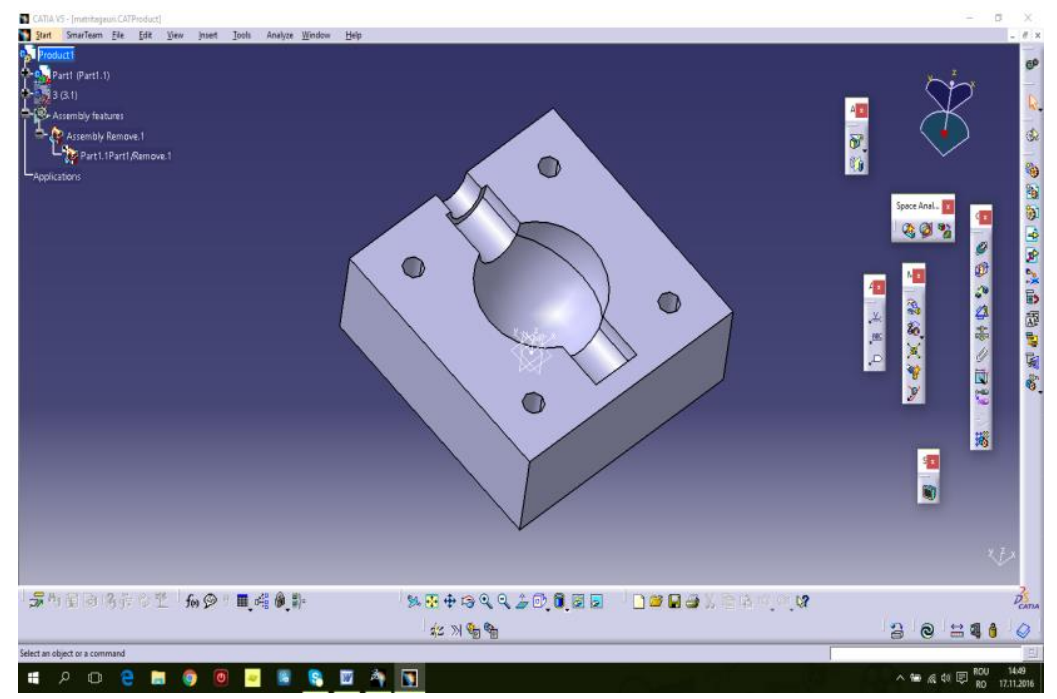

Figure 2. The 3D CAD model of the half mold. 
The using of additive manufacturing for prototype production of moulds

The manufacturing comprises three main stages: the charger one for loading the CAD model and code providing for the FDM device; the model

manufacturing stage; the third one when the model is taken away from the basic layer of the part [1][2][5][6][7][8][9].

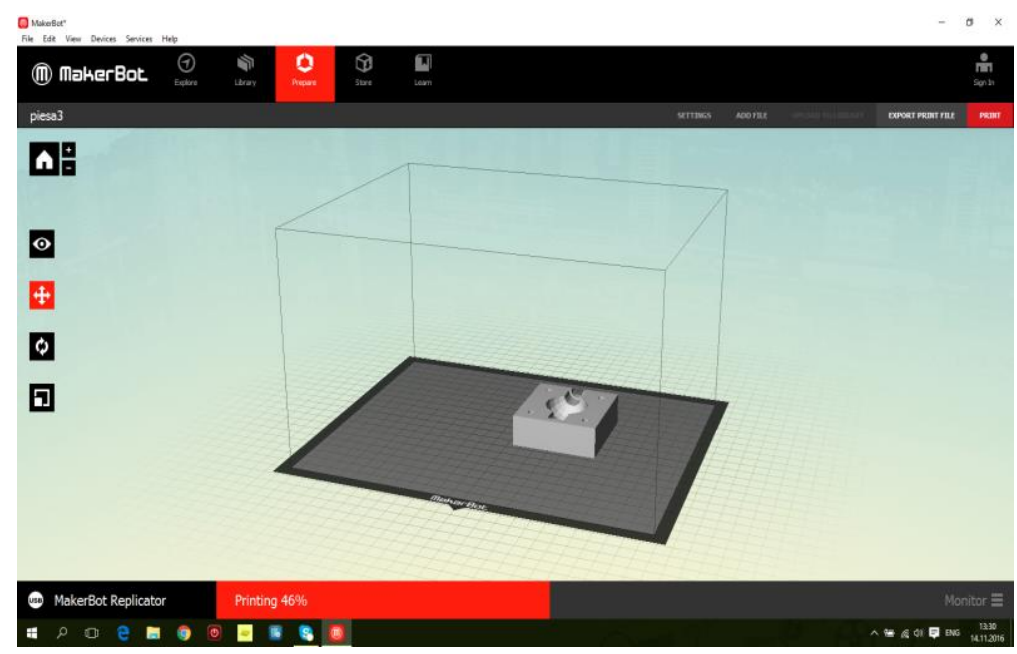

Figure 3. The before processing stage and loading of CAD model for the printer MakerBot Replicator software
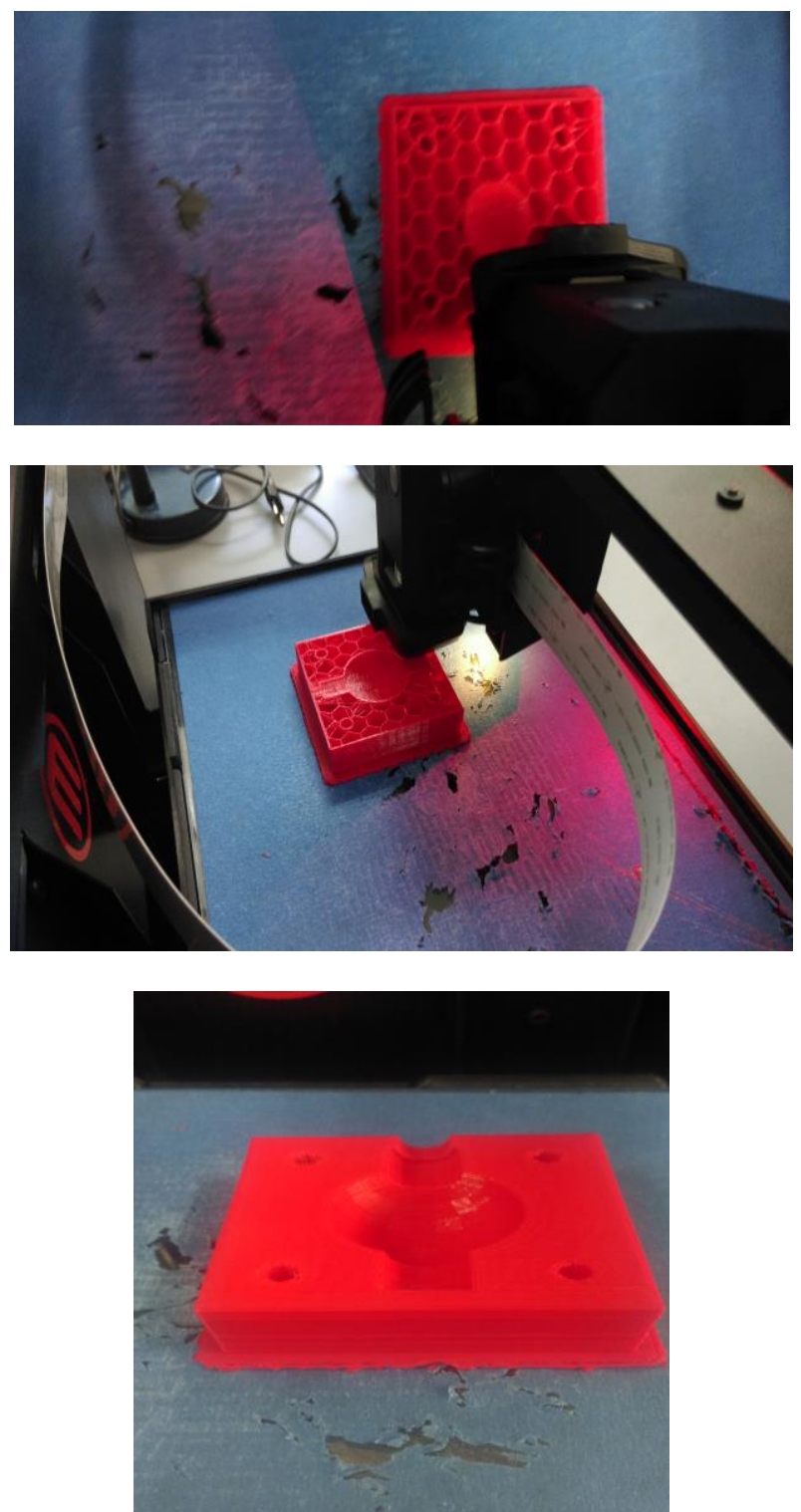

Figure 4. The printing stage and the half mold manufactured on the basic layer 
The using of additive manufacturing for prototype production of moulds

For manufacturing both the half molds, we have used the 3D printer, MakerBot Replicator with PLA material having the wire diameter $1.75 \mathrm{~mm}$ and the nozzle diameter of $0.2 \mathrm{~mm}$ as well as the section step of $0.2 \mathrm{~mm}$. The working temperature is $215^{\circ} \mathrm{C}$. The parts are placed so that the material waste for the basic layer and the dimension along the $\mathrm{OZ}$ axis should be minimum.[7][8]

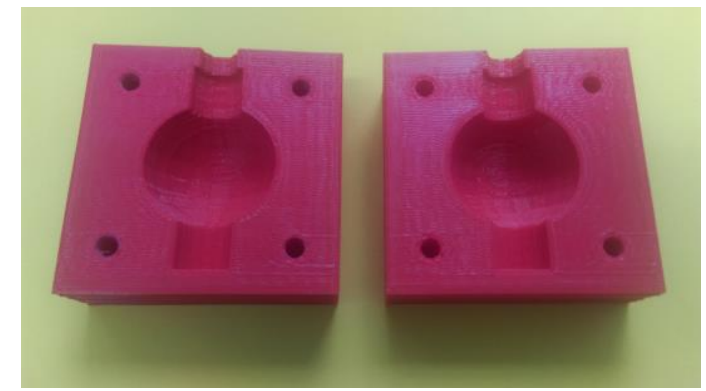

Figure 5.The two half moulds manufactured.

\section{Experimental Results}

The moulds manufactured by FDM process and made of PLA could be used directly for injection of low fusible material too, if there are not strictly imposed some other requirements regarding the material and surface qualities (Fig. 6).

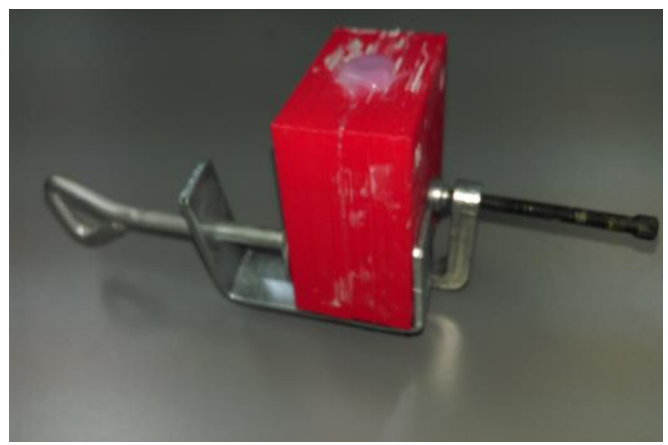

Figure 6. The assembly of both molds.

The dimensional errors are inside the range of \pm 0.2 $\mathrm{mm}$ as absolute values, meaning that if there are some additional conditions regarding the surface quality a supplementary finishing process is required. Due to the complexity of cavity shape we have to do it by hand making, so some undesired dimensional or shape changes could appear.
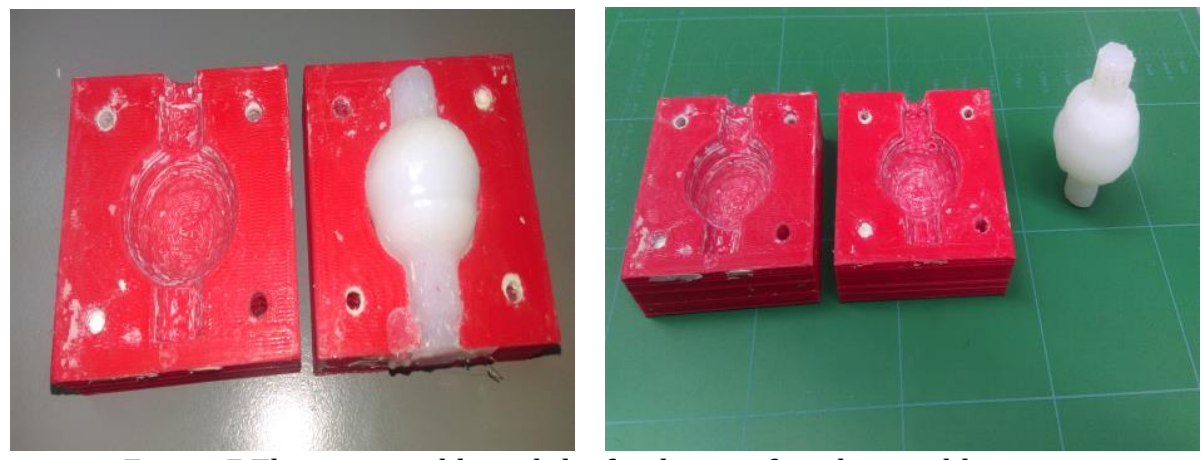

Figure 7.The two moulds and the final part after the mould process.

The manufacturing of moulds by Additive Material technology, such as FDM is another reliable way for manufacturing some parts with complex shapes. It could be applied for automotive industry, or for small series moulds, fig. 8, if the classical solutions could not be reached because of their higher prices. These new technologies provide solutions for increasing the competitive level in industry, for reducing the costs and time of the process needed for medium level of the part design.

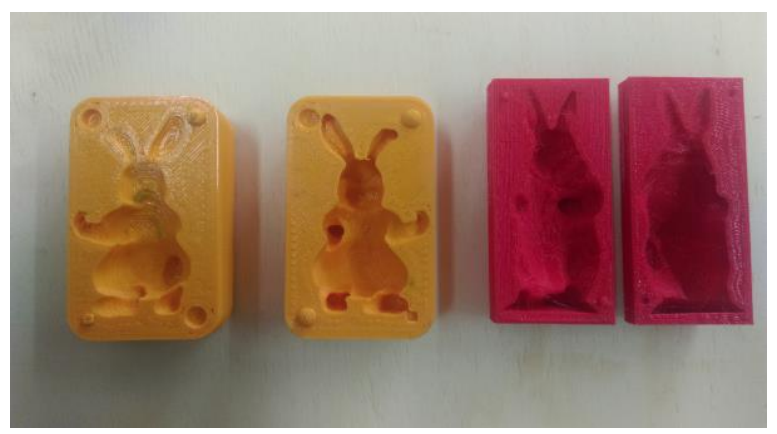

Figure 8.The manufacturing of the moulds for silicon models

\section{Conclusions}

The moulding process includes the sequence of operations implying liquid state of metal or alloy casting inside a cavity by filling it, in order to be coppied keeping its shape and finally solidiying the material, so that the new object is a result of using the model with one or more cores.An important stage of technology is the operation of model manufacturing that should be the negative of the mould and it will replicate the external surface of the part.

If the future parts, having some cavities and starting from their first stage of liquid metal that is going to poured, they have to be manufactured by using cores made of particular mixtures characterised by high resistance and hardness affording the operation of putting them inside the mould.

The permanent used cores should be made of wood, metal, plastics, composite materials, so the temporary used one should be made of industrial 
The using of additive manufacturing for prototype production of moulds

wax, paraffin or some other materials with lower melting point.

Starting with the three-dimensional model by using CAD, without any supplementary design procedure and classic cutting technologies, we may manufacture a great variety of spatial shapes even with cavities, cheaper, quickly and very accurate applying the additive manufacturing.

The problems arising from low level fusible models by moulds injection could be:

- The long period for model cooling due to the lower thermal conductivity of the material the moulds are made of.

- The stuck material which is sticked along the walls of moulds with rough surfaces.

- The material could enter inside the medium plane of the moulds due to the planarity deviation of both the mould surfaces.

The researches and experimental tests that have been done, have pointed out that the manufacturing of moulds for low fusible material injection is affordable and has some important advantages:

- the time for process preparing is not influenced by the part complexity;

- the dimensional accuracy of the moulds is good and the relative errors are lower than $1 \%$;

- if there are not imposed some special conditions regarding the quality of model surfaces, the FDM manufactured moulds may be used for injections without supplementary finishing stages for active surfaces.

\section{References:}

[1] Petru Berce, Nicolae Balc, Cristian Caizar, Răzvan Păcurar, Adrian Sever Radu, Sorin Brătean, Ioan Fodorean, Tehnologii de fabricaţie prin adăugare de material şi aplicaţiile lor, Editura Academiei Romane, București, 2014;

[2] Berce P., Balc N., Ancau M., Comsa S., Caizar C., Chezan, H., Fabricarea rapidă a prototipurilor, Editura Tehnică, Bucureşti, 2000;

[3] Bogatu L., Besnea D., Alexandrescu N., Ionaşcu G., Băcescu D., Panaitopol H., Theoretical and constructive aspects regarding small dimension parts manufacturing by stereophotolithography, Recent Advances in Mechatronics, 2007;

[4] Bogatu L., Besnea D., Ionaşcu G., Microstereophothography, a new 3D microfabrication process, Acta Technica Napocensis, nr. 49 vol. III, 2006;

[5] Mircea Mihail Popovici, Modelarea virtuală 3D, în construcţia de maşini, Bucureşti,2005;

[6] Daniel Besnea, Octavian Donţu, Nicolae Alexandrescu, Gheorghe I. Gheorghe, Paul Beca, Aurel Abalaru, Tehnologii de fabricaţie asistate de calculator pentru execuţia unor componente mecatronice, Editura Printech, Bucureşti, 2008;

[7] Daniel Besnea, Mihai Avram, Victor Constantin, Alina Spânu, Chivu Loris, Additive micromanufacturing technologies for composite materials based on nanotubes and polymers, Romanian Review Precision mechanics, optics \& mechatronics, nr. 50 / 2016;

[8] Besnea D., Spanu A., Dontu O., Gheorghe I.Gheorghe, Up - date modeling technology with thermoplastic extrusion applied for mechatronics assembly manufacturing, Romanian Review Precision Mechanics, Optics \& Mechatronics, Nr. 45 / 2014, pp.27-31;

[9] Constatin Bucsan, Mihai Avram, Daniel Besnea, Experimental models of one way valves made using rapid prototyping, Romanian Review Precision Mechanics, Optics \& Mechatronics, Nr. 48/ 2015, pp.232-236; 